\title{
Increasing Spontaneous Combustion Risk While Reducing the Coal Particle Size
}

\author{
Vyacheslav Portola ${ }^{1}$, Hi Un Lee ${ }^{2}$, Denis Botvenko ${ }^{2}$, Vladimir Ludzish², and Arhip Pavlov², \\ ${ }^{1}$ T.F. Gorbachev Kuzbass State Technical University, 650000, Kemerovo, Russian Federation \\ ${ }^{2} \mathrm{PC}$ «Scientific Center VostNII», 650000, Kemerovo, Russian Federation
}

\begin{abstract}
Extraction, storage and transportation of coal are complicated by the danger of spontaneous combustion centers due to toxic gases emission. At various technological processes, the formation of clusters consisting of coal particles of various sizes is possible. In the course of the research, the influence of the size of coal particles on the danger of spontaneous combustion of oxidizing material clusters was estimated. The studies of coal chemical activity have shown that for most seams of Kuzbass mines, the amount of absorbed oxygen increases significantly with decreasing particle size. Coal dust can be more active than coal in 3-8 times. For some seams, the coal breakage has almost no effect on its chemical activity in relation to oxygen. It is determined that the duration of incubation period of spontaneous combustion also varies depending on the size of particles forming the coal cluster. For the appearance of spontaneous combustion centers, the size of coal dust clusters may be in 2 4 times smaller than for coal. In order to increase the safety of work for all the seams it is necessary to study the chemical activity in relation to oxygen, the tendency to spontaneous combustion, and the duration of the incubation period not only for coal samples, but also for coal dust.
\end{abstract}

\section{Introduction}

Coal is a substance that can interact with atmospheric oxygen at natural ambient temperatures. The accession of oxygen occurs with the emission of heat, which under certain conditions can cause the development of spontaneous combustion process. Considering that spontaneous combustion is a source of toxic gases, they can initiate explosions of a combustible gas; many studies have been devoted to the research of this process [1-6]. The danger of emission of various gases [7, 8] and the dependence of the process on various factors $[3,9-12]$ were estimated. One of the significant factors affecting the possibility of spontaneous combustion is the chemical activity of coal, corresponding to the volume of oxygen absorbed by the coal mass per unit of time. Under the condition of a constant air flow to the clusters of oxidizing substance, the amount of heat released from the coal is proportional to its chemical activity. Therefore, the possibility of endogenous fires significantly increases in pits when mining seams with high chemical activity, as well as in the subsequent storage, processing and transportation of such coal.

According to Russian regulations, it is necessary to determine the propensity for spontaneous combustion and, accordingly, the chemical activity of coal in relation to 
oxygen for all newly mined seams and to repeat such studies every three years. However, existing methods provide such measurements for coal samples consisting of 1-3 mm particles in size. Meanwhile, the introduction of powerful coal mining technology has led to an increase in emission of coal dust, the particle size of which is less than $1 \mathrm{~mm}$. A significant amount of coal dust enters the worked-out space of excavation areas, where the clusters of coal dust are being formed and the subsequent development of the spontaneous combustion process is possible.

An important role in the formation of coal dust clusters in mines is played by air leaks in the mined-out space, capable of carrying particles of coal dust over long distances. Due to the heterogeneity of the space developed, the cross-sectional area of the channels through which air moves with dust particles may vary. Therefore, the dust fall in the developed part of the seam occurs unevenly, and the maximum amount of coal particles falls in the widest places of channels, in which the air velocity decreases sharply. The fractional composition of dust accumulated in the developed space also changes as the air flow removes from the face. Near the face in the developed space large particles will fall, and as the distance from it increases, the size of the precipitated coal particles decreases. However, due to the constant face movement, the accumulated coal dust clusters will be represented by different fractions.

The main amount of coal dust gets into the air streams, including leaks, entering the mined-out space, directly from the working body of the coal-mining combine. The highest air permeability of the seam's worked-out part is observed along the pillars of the extracted coal column; therefore, along the contours of the worked-out space, we should expect the formation of coal dust clusters. The part of generated dust settles in the existing mine workings; however, as the mining face moves, this dust also appears in the developed space and can be redistributed by flows of filtered air.

One of the likely places of coal dust clusters' formation is the degassing wells in the worked-out space. When air flows from the channels being formed in the loosened mass of coal and rocks to the well, a sharp drop in gas velocity and sedimentation of dust particles may occur. The coal dust clusters formed under the wells are provided with a constant air flow, which leads to oxidation of combustible material and may contribute to the spontaneous combustion. The possibility of spontaneous combustion and the rate of temperature increase in the oxidizing substance depend on the velocity of the filtered air, the oxygen concentration in it, the size of the cluster and its thermo physical parameters.

The possibility of formation of coal dust clusters also exists at other mining enterprises using coal. Thus, a significant amount of coal dust occurs at the processing plants in the process of crushing, sorting and transporting coal. To improve the efficiency of burning coal is also crushed and moved to the furnace in the form of coal dust. Practice shows that spontaneous combustion centers that can initiate explosions of combustible gas and coal dust can be formed in coal dust clusters existing at these enterprises.

\section{Results and Discussion}

Considering the increasing possibility of coal dust clusters' formation, it is necessary to evaluate the change in the chemical activity of coal of various seams with decreasing particle size, as well as the possibility of developing a spontaneous combustion process in such coal dust clusters. To assess the chemical activity of samples of coal and coal dust, the oxygen sorption rate constant of coal was used, which represents the amount of gas absorbed by coal placed in pure oxygen. Coal was taken from various seams of Kuzbass mines, then in the laboratory, these samples were crushed and scattered into various fractions. Coal was represented by particles $1-3 \mathrm{~mm}$ in size, and samples with a particle size of 0.2-1.0 mm, 0.064-0.2 $\mathrm{mm}$ and less than $0.064 \mathrm{~mm}$ were used as coal dust. 
Selected samples of coal and coal dust were simultaneously placed in sorption containers, sealed and kept for 24 hours. Then, the remaining oxygen concentration in the container atmosphere was determined, and the rate of oxygen sorption by coal was calculated using the formula

$$
U=-\frac{V}{M \tau} \ln \frac{C_{A}\left(1-C_{0}\right)}{C_{0}\left(1-C_{A}\right)}
$$

where $V$ is the volume of air in contact with coal, $\mathrm{cm}^{3} ; M$ is the mass of the sample of coal, g; $\tau$ is the time of air contact with coal, h. $C_{0}$ is the initial oxygen concentration in the container, unit fraction; $C_{A}$ is oxygen concentration in time $\tau$, unit fraction.

The results of the study of oxygen sorption rate constant by coal of several seams from Kuzbass mines, depending on the size of their constituent particles, are given in Table 1.

Table 1. Change of oxygen sorption rate constant by coal $(\mathrm{ml} /(\mathrm{g} \cdot \mathrm{h}))$ depending on particle size.

\begin{tabular}{|l|c|c|c|c|}
\hline \multirow{2}{*}{ Coal seam } & \multicolumn{4}{|c|}{ Size of coal particles in samples, mm } \\
\cline { 2 - 5 } & $\mathbf{1 - 3}$ & $\mathbf{0 . 2 - 1 . 0}$ & $\mathbf{0 . 0 6 4 - 0 . 2}$ & $<\mathbf{0 . 0 6 4}$ \\
\hline Coal seam 15 & 0.0697 & 0.1113 & 0.1203 & 1.1135 \\
\hline Coal seam 7-7a & 0.0365 & 0.0658 & 0.0691 & 0.0673 \\
\hline Coal seam 26a & 0.0433 & 0.0561 & 0.499 & 0.0523 \\
\hline Coal seam Bezymyanny & 0.1730 & 0.2053 & 0.6645 & 1.4027 \\
\hline Coal seam Sychevsky IV & 0.2354 & 0.5646 & 1.1997 & 1.3645 \\
\hline Coal seam Gramoteinsky II & 0.1351 & 0.3473 & 0.7586 & 0.4871 \\
\hline Coal seam E-5 & 0.0904 & 0.1152 & 0.0788 & 0.1037 \\
\hline
\end{tabular}

The analysis of experiment results shows that reducing the particle size to a state of dust ambiguously affects the chemical activity of various coal seams. In some seams, samples of coal and coal dust have almost identical chemical reactivity values in relation to oxygen. But for most seams, dust samples are much more active than coal samples. So, coal breakage in E-5 and 26a seams, practically does not change its chemical activity. For coal seam 7-7a, a sharp increase (almost twice) in chemical activity with a decrease in particle size from $1-3 \mathrm{~mm}$ to $0.2-1.0 \mathrm{~mm}$ is typical, and a further decrease in the size of coal particles practically does not change their ability to absorb oxygen.

Most of the coal seams (seams 15, Bezymyanny, Sychevsky IV and Gramoteinsky II) show a significant increase in chemical activity with a decrease in particle size. For example, the coal dust of seam Bezymyanny with a size of less than $0.064 \mathrm{~mm}$ has a chemical activity in 8 times greater than a sample of coal with a particle size of 1-3 mm. The activity of the smallest coal dust of seam 15 increases almost in 16 times in comparison with samples of coal having a particle size of 1-3 $\mathrm{mm}$. Some peculiarity in this group has the coal of seam Gramoteinsky II, which demonstrates a decrease in chemical activity for dust with a particle size of less than $0.064 \mathrm{~mm}$.

One of the criteria for assessing the danger of the development of spontaneous combustion process in coal clusters is the duration of spontaneous combustion incubation period, which is the time during which the cluster heats up to a critical temperature. Most of the coals have a critical temperature of $80-110^{\circ} \mathrm{C}$, reaching which the heating process becomes irreversible. To assess the effect of breakage on the speed of heating of the oxidizing material, we calculate how the duration of incubation period of spontaneous combustion of coal and coal dust clusters with particles of different sizes changes. The duration of incubation period of coal spontaneous combustion (in days) can be determined by the formula 


$$
\tau_{\text {ink }}=\frac{c\left(T_{\kappa}-T_{0}\right)+0.6 j W+q_{d} X}{24 \alpha U^{0,45} C_{1} q},
$$

where $c$ is the heat capacity of coal, cal / $(\mathrm{g} \cdot \mathrm{K}) ; T_{K}$ is the critical temperature of coal spontaneous combustion, $\mathrm{K} ; T_{0}$ is the initial temperature of coal, $\mathrm{K} ; j$ is the heat of water evaporation, cal / $\mathrm{g} ; W$ is the initial moisture of coal, unit fraction; $q_{d}$ is the specific heat of methane desorption, cal / $\mathrm{ml} ; X$ is natural coal gas content, $\mathrm{ml} / \mathrm{g}$; $\alpha$ is the oxygen absorption coefficient of the air; $U$ is the oxygen sorption rate constant of coal, $\mathrm{ml} /(\mathrm{g} \cdot \mathrm{h})$; $C_{l}$ is the volume concentration of oxygen at the entrance to the coal cluster, unit fraction; $q$ is the specific heat of sorption of air oxygen by coal, cal / $\mathrm{ml}$.

The analysis of formula (2) shows that the duration of incubation period of coal spontaneous combustion decreases significantly with increasing chemical activity of the oxidizing material, as well as with decreasing moisture and natural gas content in the coal sample. The duration of the incubation period was calculated for the coal and coal dust clusters of several seams, given in Table. 1. The calculated values of chemical activity of the samples in relation to oxygen were used as initial data for the calculations. The critical temperature of spontaneous combustion was $90^{\circ} \mathrm{C}$. The remaining parameters (humidity, natural gas content of coal) remained unchanged for all samples. The results of the calculations are given in Table 2

Table 2. The duration of incubation period of coal spontaneous combustion (day) depending on the particle size of the cluster.

\begin{tabular}{|l|c|c|c|c|}
\hline \multirow{2}{*}{ Coal seam } & \multicolumn{4}{|c|}{ Size of coal particles in the cluster, mm } \\
\cline { 2 - 5 } & $\mathbf{1 - 3}$ & $\mathbf{0 . 2 - 1 . 0}$ & $\mathbf{0 . 0 6 4 - 0 . 2}$ & $<\mathbf{0 . 0 6 4}$ \\
\hline Coal seam 15 & 57.0 & 46.2 & 44.6 & 16.3 \\
\hline Coal seam 7-7a & 77.0 & 58.7 & 57.0 & 57.8 \\
\hline Coal seam Bezymyanny & 37.7 & 34.9 & 20.6 & 14.7 \\
\hline Coal seam Sychevsky IV & 32.8 & 22.1 & 15.8 & 14.9 \\
\hline Coal seam Gramoteinsky II & 42.2 & 27.6 & 18.8 & 23.7 \\
\hline
\end{tabular}

Evaluating data given in Table 2, we can conclude that the duration of development of spontaneous combustion process in coal dust clusters is sharply reduced for many seams compared to usual coal clusters. Thus, the duration of coal dust spontaneous combustion from seam 15 is in 3.5 times less than that of coal. Therefore, at the stage of estimating the tendency of coal to spontaneous combustion and determining the duration of its incubation period, it is necessary to conduct studies of coal dust. If there is a danger of coal dust clusters formation in the mined-out space, it is necessary to focus on the magnitude of the chemical activity and the duration of incubation period of spontaneous combustion determined for dust.

An important parameter characterizing the possibility of spontaneous combustion of an oxidizing material is the critical size of the cluster necessary for fire breaking-out. As a critical size, a minimum radius or diameter of the ball is used, at which heat in an oxidizing cluster is emitted more than is lost to the surrounding space, which increases the temperature of the cluster. The critical size of coal cluster depends on the ratio of the heat generated in the volume of coal due to its oxidation, and the heat lost from the heated volume into the surrounding space.

The amount of heat generated in the volume of oxidizing material cluster having a spherical shape can be determined by the formula

$$
Q_{1}=\frac{4 \pi}{3} r^{3} q C \rho U
$$


where $\mathrm{r}$ is the radius of the oxidizing substance cluster, $\mathrm{m}$; $\mathrm{C}$ is the volume concentration of oxygen in the air entering the cluster, unit fraction; $p$ is the density of coal cluster, $\mathrm{kg} / \mathrm{m} \mathrm{M}^{3}$.

The amount of heat lost by coal cluster at the boundary of the ball can be written in the following way

$$
Q_{2}=\pi 4 r^{2} \lambda \frac{d t}{d r}
$$

where $t$ is the temperature, $K ; \lambda$ is the thermal conductivity coefficient of coal cluster, $\mathrm{W} /(\mathrm{m} \cdot \mathrm{K})$.

For the case of steady equality between generated and lost heat, we equate equations (3) and (4), divide the variables and integrate the obtained equality under the boundary conditions

$$
\left.t\right|_{r=0}=T_{0} ;\left.\quad t\right|_{r=R}=T_{1}
$$

where $T_{0}$ is the temperature in the center of spontaneous combustion cluster, $\mathrm{K} ; \mathrm{T}_{1}$ is the natural temperature of rocks, $\mathrm{K}$,

we obtain a formula for determining the radius of the heated zone

$$
R=\sqrt{\frac{6 \lambda\left(T_{0}-T_{1}\right)}{C q \rho U}}
$$

Using the data of speed constant for the sorption of oxygen by coal and coal dust given in Table 1, the critical radii of clusters were calculated, sufficient to reach a critical temperature of $90^{\circ} \mathrm{C}$. The heat transfer coefficient of coal and dust in the calculations was $0.2 \mathrm{~W} /(\mathrm{m} \cdot \mathrm{K})$, the density of clusters is $1200 \mathrm{~kg} / \mathrm{m}^{3}$, the ambient temperature is $20^{\circ} \mathrm{C}$. The calculation results are shown in Table 3

Table 3.The critical radius of coal and coal dust clusters $(\mathrm{m})$,providing a temperature rise to a critical value.

\begin{tabular}{|l|c|c|c|c|}
\hline \multirow{2}{*}{ Coal seam } & \multicolumn{4}{|c|}{ Size of coal particles in the samples, mm } \\
\cline { 2 - 5 } & $\mathbf{1 - 3}$ & $\mathbf{0 . 2 - 1 . 0}$ & $\mathbf{0 . 0 6 4 - 0 . 2}$ & $<\mathbf{0 . 0 6 4}$ \\
\hline Coal seam 15 & 0.63 & 0.50 & 0.48 & 0.16 \\
\hline Coal seam 7-7a & 0.87 & 0.65 & 0.63 & 0.64 \\
\hline Coal seam Bezymyanny & 0.40 & 0.36 & 0.20 & 0.14 \\
\hline Coal seam Sychevsky IV & 0.34 & 0.22 & 0.15 & 0.14 \\
\hline Coal seam Gramoteinsky II & 0.45 & 0.28 & 0.19 & 0.24 \\
\hline
\end{tabular}

The data given in Table 3 indicate that the development of spontaneous combustion process in coal dust requires the formation of clusters of much smaller size than for coal. It should be taken into account that as the temperature of coal increases, its chemical activity will also increase, so under real conditions the thickness of the coal dust layer in which the spontaneous combustion center may develop will be significantly less than the calculated values.

\section{Conclusions}

Analyzing the results of the research, we can make the following conclusions.

1. Most of the studied coal seams produce coal dust, the chemical activity of which in relation to oxygen is significantly higher than that of coal. 
2. The incubation period of spontaneous combustion of coal dust can be reduced in 2-4 times compared with coal.

3. For the development of spontaneous combustion process in coal dust, the formation of clusters of much smaller size than for coal is required.

4. For all the seams being worked out it is necessary to study the chemical activity in relation to oxygen, the tendency to spontaneous combustion and the duration of incubation period, not only for coal samples, but also for coal dust.

5. The obtained results should be taken into account for assessing the danger of development of spontaneous combustion process in cases where the formation of coal dust clusters during the extraction, enrichment, transportation and use of this coal is possible.

\section{References}

1. Q. Lin, S. Wang, S. Song, Y. Liang, T. Ren, Fuel processing Technology, 159, 38 (2017)

2. S. C. Banerjee, Spontaneous Fire Risk Estimation and its Prevention. Prevention and combating Mine Fires (Mining, Prague, 2000)

3. V.A. Portola, Journal of Mining Science, 32:6, 536 (1996)

4. M.N. Tarafadar, D. Guha, Fuel, 68, 315 (1989)

5. J.W. Weier, Fire in Hole. Sciences, 37:6, 12 (1997)

6. D.S. Nimaje, D.P. Tripathy, The Indian Mining \& Engineering Journal, 10 (2010)

7. V. A. Portola, Radon Emission from Coal Mines of Kuzbass Region, 127, 5 (2016)

8. V.A. Portola, Journal of Mining Science, 32:3, 212 (1996)

9. J. Deng, J.-Y. Zhao, Y.-N. Zhang, C.-R. Wang, A.-C. Huang, C.-M. Shu, Journal of Thermal Analysis, 127:1, 439 (2017)

10. L. Zhang, B. Qin, Fire and materials, 40:2, 246 (2016)

11. A. Rosema, Y. Guan, H. Veld, Article in Fuel, 80:1, 7 (2001)

12. Khi Un Lee, The development of a control method for aerogasdynamic processes in the mine workings of carbon dioxide mines (CMIAS, Beijing, 2003) 